by the sobering influence of experience. Females, representing fewer than a quarter of those in the series, are less exposed to crushing trauma of the hands. There was an almost equal distribution of injuries between the left and right hands, but only one of the 14 fractures involved a digit of the left hand. Thus it would appear that the injuries to the right hand are slightly more common and in general more severe than those to the left hand.

The incidence of fractures of the terminal phalanx as shown on routine $x$-ray examination of all cases was $19 \%$. This is in keeping with the findings of Iselin (1940), who observed such fractures in a quarter of his cases. The post-operative infection of the subungual space which occurred in three cases responded rapidly to parenteral antibiotic therapy.

Tenderness was present for an average of 14.3 days in an uncomplicated case, but this period was lengthened by 2.5 days in the presence of a fracture of the terminal phalanx. For the last nine days of treatment protective strapping prevented further trauma and immobilized the injured digit.

That $81 \%$ of patients subsequently lost the nail would tend to support the view of Robins (1961) that if the nail is floating it becomes a foreign body and should be removed. Certainly this rate of nail loss is very high, but it seems reasonable to suggest that rather than remove the nail at the first visit, leaving a raw painful surface, it should be left attached but decompressed by the method which is most likely to lead to its being retained and continuing to grow.

The results in this series illustrate that the initial size of the haematoma provides no indication of the likelihood of the subsequent loss of the nail (see Table II) ; there may even be an underlying fracture of the terminal phalanx with only a small haematoma to be seen. If treatment is begun within 48 hours of the injury there is approximately a $20 \%$ chance that the nail will survive. This chance is increased in the younger patient by virtue of age.

If a comparison is made of the various methods of decompression it will be seen that only $10.3 \%$ of those cases in which incision through the nail-bed was used retained the nail, and tenderness was present for 14 days. When a distal incision beneath the nail-tip was made, $16.6 \%$ of nails subsequently survived, tenderness again lasting for 14 days. The method of choice, therefore, must be decompression by means of a red-hot wire, which gave a nail-retention rate of $28.6 \%$ and a duration of tenderness of 12 days. Of the cases in which the nail was subsequently lost, $5(7 \%)$ patients regarded the newly growing nail as abnormal in shape, but in no instance was there a complete failure of nail regeneration.

Provided local digital-block analgesia is used, so that the effusion may be completely expressed and an absorptive dressing applied to exert a continuous wick action, the hot-wire method offers the best prospect of retention of the nail with the earliest freedom from pain.

\section{Summary}

In this survey 72 patients attending a casualty department for treatment of a subungual haematoma have been investigated. All relevant information regarding the injury, the treatment given, and the progress made was recorded, and subsequently the relative merits of decompression by three methods were assessed.

Subungual haematoma is a relatively common injury, an average of three cases a week being seen in this department.

An associated fracture of the terminal phalanx was seen on routine $x$-ray examination in $19 \%$ of these cases.

The initial size of the haematoma gives no reliable indication of the presence or absence of an underlying fracture.

Decompression within 48 hours of the injury increases the likelihood that the nail will survive and continue to grow.

The results of this survey suggest that the highest percentage of patients $(28.6 \%)$ retain the nail involved, and are free from pain in the shortest time after decompression of the effusion by penetration of the proximal part of the nail with a red-hot wire under local analgesia.

I am indebted to $\mathrm{Mr}$. M. Ellis, consultant in charge, the receivingroom, the General Infirmary at Leeds, for his permission to carry out this survey and for his advice; to Professor J. C. Goligher for reading the manuscript; and to the staff and students of the receiving-room for their help in making this investigation possible.

\section{REFERENCES}

Ellis, M. (1962). The Casualty Officer's Handbook, p. 121. Butterworth,

Flemming, C. (1946). Minor Surgery, 23rd ed., p. 79. Churchill, London.

Iselin, M. (1940). Surgery of the Hand. Churchill, London.

Robins, R. H. C. (1961). Injuries and Infections of the Hand, p. 115. Arnold, London.

Wright, A. D. (1952). In Practical Procedures, edited by Sir Heneage Ogilvie and W. A. R. Thomson, p. 57. Eyre and Spottiswoode, I,ondon.

\title{
Acute Leukaemia Associated With Phenylbutazone Treatment
}

\author{
H. J. WOODLIFF,* M.B., PH.D., M.R.C.P.ED. ; LESLEY DOUGAN,†, M.B., B.S., B.SC.
}

Brit. med. F., 1964, 1, 744-746

Phenylbutazone, widely used in the treatment of rheumatic disorders, is known to cause bone-marrow depression in some patients. It has also been suggested that this drug might be associated with leukaemia. The first report concerned six patients who developed leukaemia after phenylbutazone treatment (Bean, 1960). Study of the case histories reveals that these patients did not all fall clearly into the classical patterns of the well-known types of leukaemia. Nevertheless,

\footnotetext{
$\star$ Head of the Department of Haematology, Royal Perth Hospital, Perth Western Australia.

† Research Fellow, Cancer Council of Western Australia.
}

these cases could be regarded as malignant proliferations of leucopoietic tissue. Cast (1961) described a case of chronic myeloid leukaemia after the ingestion of $210 \mathrm{~g}$. of phenylbutazone, and Cadman and Limont (1962) reported a case of acute leukaemia in which symptoms began 11 weeks after a short course of phenylbutazone.

In April 1959 a registry of leukaemia and allied disorders was set up in Western Australia. By June 196355 cases of acute leukaemia diagnosed in this period had been reported to the registry, and in most of these a history of drugs taken before the disease developed was sought. Of these patients, 
eight gave a history of phenylbutazone ingestion before the onset of the disease. Radiotherapy had also been given to two of them, and a third received phenylbutazone only a short time before the diagnosis was made. In five, however, no other known leukaemogenic agents were implicated, and the period of ingestion before the development of the disease was such as might be expected if the drug was concerned in the development of the disorder.

Among 417 other cases of chronic leukaemia, lymphoma, and allied disorders notified to the registry, five were found with a history of phenylbutazone ingestion. These were two cases of Hodgkin's disease, one of chronic lymphatic leukaemia, and two of polycythaemia vera.

The case histories of the five patients with acute leukaemia are presented here.

\section{Case 1}

The patient, a male born in 1884, died of acute myeloblastic leukaemia in August 1962. He had transurethral resection performed in 1961, when a well-differentiated adenocarcinoma of the prostate was found; at this time the peripheral blood findings were unremarkable. In May 1962 he was admitted to the Royal Perth Hospital for investigation of back pain. He gave a history of arthritis for 30 years and he had been treated with phenylbutazone since 1958, taking $300 \mathrm{mg}$. daily in intermittent courses. The latest course had been a few weeks before admission.

On examination, pallor and hepatomegaly were noted and small soft lymph nodes were palpable in the left axilla and groins. Peripheral blood examination showed haemoglobin $8 \mathrm{~g} . / 100 \mathrm{ml}$., white-cell count $1,600 / \mathrm{c} . \mathrm{mm}$. (polymorphs $55 \%$, lymphocytes $42 \%$, monocytes $3 \%$ ), and platelets $78,000 / \mathrm{c} . \mathrm{mm}$. Marrow aspiration from the manubrium sterni resulted in a blood tap; using a Turkel needle, a specimen was obtained from the iliac crest. No fragments were obtained and smears of the aspirate were only moderately cellular. There was an increased proportion of blast cells $(30 \%)$, which cytochemical studies proved to be myeloblasts. A diagnosis of aplastic anaemia was considered, but after a review of the available material this was rejected in favour of a diagnosis of acute myeloblastic leukaemia. He was treated with corticosteroids and blood transfusion, but died three months later with what was presumed to be a cerebral haemorrhage. Necropsy was not performed. The diagnosis of acute myeloblastic leukaemia was confirmed by the Diagnostic Group of the Leukaemia and Allied Disorders Committee of the Cancer Council.

The onset of acute leukaemia in this case appears to have been associated with bone-marrow depression and occurred after a four-year period of intermittent phenylbutazone ingestion.

\section{Case 2}

The patient, a male born in 1909, died of acute lymphoblastic leukaemia in April 1962. He developed arthritis in 1955 and had five courses of phenylbutazone in daily doses of $200 \mathrm{mg}$. between then and 1962. The most recent course had been concluded in July 1961 . He was admitted to a country hospital in December 1961 for investigation of hepatomegaly, weakness, and anorexia which followed an attack of pneumonia. Blood examination showed a haemoglobin of $5.2 \mathrm{~g} . / 100 \mathrm{ml}$. and a white-cell count of $122,000 /$ c.mm. A diagnosis of leukaemia was made and he was transfused prior to transfer to Royal Perth Hospital.

On admission a blood count showed: haemoglobin 8.8 g./100 $\mathrm{ml}$. ; white-cell count $105,000 /$ c.mm. ; the majority of leucocytes were leukaemic lymphoblasts. A bone-marrow examination revealed a cellular marrow. Over $90 \%$ of the cells present were leukaemic lymphoblasts. A diagnosis of acute lymphoblastic leukaemia was made and the patient was treated with blood transfusions and prednisolone. After a short remission relapse occurred and he was treated with mercaptopurine. In spite of this he died in April 1962.

The onset of acute lymphoblastic leukaemia occurred in this case some five months after phenylbutazone ingestion and there was a six-year history of intermittent treatment with phenylbutazone.

\section{Case 3}

The patient, a male born in 1880, died of acute myeloblastic leukaemia in January 1961. He was admitted to Royal Perth Hospital in October 1960 with chest pain, weakness, dizziness, pallor, and easy bleeding. He had had arthritis for five years and had taken phenylbutazone $300 \mathrm{mg}$. daily for five days of each week from 1956 until October 1960.

Examination showed marked pallor and hepatomegaly. The haemoglobin was 8 g. $/ 100 \mathrm{ml}$., white-cell count 4,700/c.mm. (blasts $3 \%$, premyelocytes $6 \%$, myelocytes $2 \%$, metamyelocytes $2 \%$, polymorphs $40 \%$, lymphocytes $36 \%$, monocytes $11 \%$ ), and platelets $57,000 /$ c.mm. There were 65 normoblasts per 100 white cells. Marrow examination showed a reduction in granulocytes and megakaryocytes; cells were mainly of the erythroid series and many showed abnormalities in shape and staining reaction of the nuclei. A diagnosis of erythroleukaemia was made and he was treated with repeated blood transfusions. In December 1960 the blood picture became more suggestive of acute myeloid leukaemia. The haemoglobin was 7.8 g. $/ 100 \mathrm{ml}$., white-cell count $4,300 /$ c.mm. (blasts $5 \%$, premyelocytes $11 \%$, myelocytes $21 \%$, metamyelocytes $3 \%$, polymorphs $24 \%$, lymphocytes $28 \%$, monocytes $1 \%$, eosinophils $2 \%$, basophils $5 \%$ ), and platelets 7,000/c.mm. He died in January 1961. Post-mortem findings were consistent with the diagnosis of acute myeloblastic leukaemia.

The onset of leukaemia in this case occurred after ingestion of $1,500 \mathrm{mg}$. of phenylbutazone weekly for four years.

\section{Case 4}

The patient, a female born in 1883, was diagnosed as having acute myeloblastic leukaemia in May 1963. She had suffered from arthritis since 1958, and began to take phenylbutazone in 1960 . During 1961 she took a daily dosage of 100 or $200 \mathrm{mg}$. and ceased therapy in April 1962. She had a history of anaemia going back to 1950 and thought to be associated with a bleeding peptic ulcer. In April 1961 she began to complain of tiring easily. In May 1962 normal peripheral blood findings were recorded. In March 1963 she was admitted to Royal Perth Hospital with anaemia. Purpura was noted and the haemoglobin was $8 \mathrm{~g} . / 100 \mathrm{ml}$. She was treated with blood transfusion. In May 1963 peripheral blood examination showed haemoglobin $10.6 \mathrm{~g} .1100 \mathrm{ml}$; white-cell count 4,000/c.mm. (neutrophils $25 \%$, lymphocytes $47 \%$, monocytes $15 \%$, blasts $12 \%$, metamyelocytes $1 \%$ ), and platelets $65,000 /$ c.mm. The appearances were considered suggestive of acute leukaemia. Marrow examination confirmed a diagnosis of acute myeloblastic leukaemia. She was treated with repeated blood transfusions and had a short course of mercaptopurine in September. Remission did not occur and she died in November 1963.

Acute myeloblastic leukaemia in this case was diagnosed some 12 months after ingestion of phenylbutazone, and the period of ingestion was for some 16 months.

\section{Case 5}

The patient, a female born in 1905, had suffered from arthritis in 1958 and had four courses of phenylbutazone between 1958 and 1963.

From March until May 1963 she suffered various pyogenic infections ; in July she was feeling generally unwell and was complaining of retrosternal discomfort and retrosternal dysphagia. She was admitted to a private hospital at this time. Peripheral blood examination showed haemoglobin $11.1 \mathrm{~g} . / 100 \mathrm{ml}$. and white-cell count $1,400 /$ c.mm. (neutrophils $8 \%$, lymphocytes $70 \%$, blasts $22 \%$ ). Platelets were scanty in the blood film. A bone-marrow examination showed a moderately cellular marrow; the majority of the cells present were leukaemic myeloblasts.

A diagnosis of acute myeloblastic leukaemia was made and she was treated with mercaptopurine, but no remission occurred and she died in August 1963.

\section{Discussion}

The previously recognized association of phenylbutazone and leukaemia with the suggestion that the drug might be 
implicated as the cause of some cases of leukaemia, receives support from the present investigation.

Before accepting a relationship of cause and effect between the drug and the disease, it is necessary to show that leukaemia occurs in treated patients in a significantly greater proportion than in the general population.

Unfortunately the number of patients treated with phenylbutazone in this community is not known, although it has been estimated that 3,000 prescriptions of the drug are filled weekly in Western Australia (W. Inglis, personal communication, 1963).

In the period under review 55 adults developed acute leukaemia and five of these had a significant history of phenylbutazone ingestion. If there is no association between the two this would suggest that $9 \%$ of the adult population was taking phenylbutazone. In spite of its widespread use, we feel that this is most unlikely and that there is a real association between acute leukaemia and phenylbutazone ingestion. Further support for this view is given by the fact that the incidence of phenylbutazone ingestion was $9 \%$ among the patients with acute leukaemia and only $1.2 \%$ among those with allied disorders.

The mechanism by which phenylbutazone may cause acute leukaemia is not known. However, in one of our patients (Case 1) it appears that the drug first depressed the bonemarrow. Bean's first case illustrates the progression from hypoplasia to leukaemia. Possibly the normal leucopoietic stimuli acting on a depressed marrow might induce a leukaemic state.

\section{Summary}

Phenylbutazone, an accepted cause of bone-marrow depression in some patients, has also been reported as being associated with leukaemia. In the present investigation 8 out of 55 patients with acute leukaemia had a history of phenylbutazone ingestion. In two cases radiotherapy had also been given and in a third the phenylbutazone was given only a short time before the leukaemia became manifest. In five cases, however, the histories are such that the drug may have been associated with the development of the disorder. This incidence of $9 \%$ is compared with an incidence of phenylbutazone ingestion of $1.2 \%$ in 417 patients with chronic leukaemia, lymphoma, and allied disorders.

The association between phenylbutazone and acute leukaemia is thus thought to be real. If there was no association then the figures suggest that $9 \%$ of the adult population of Western Australia have taken phenylbutazone.

We wish to thank those doctors who have referred their patients to the registry and the members of the Diagnostic and Epidemiology Groups of the Leukaemia and Allied Disorders Committee for their assistance in the discussion and diagnosis of these cases; Dr. W. Inglis and Dr. R. L. Kirk have also given help and advice.

\section{REFERENCES}

Bean, R. H. D. (1960). Brit. med. F., 2, 1552. Cadman, E. F. B., and Limont, W. (1962). Ibid., 1, 798. Cast, I. P. (1961). Ibid., 2, 1569.

\section{Medical Memoranda}

\section{Herpetic Erythema Multiforme}

Brit. med. F., 1964, 1, 746-747

Erythema multiforme exudativum (Hebra's disease) is an acute eruption of the skin and often of the mucous membrane. The lesions include macules, papules, vesicles, bullae, and urticarialike lesions. Sometimes the mucous membranes of the oral cavity, eyes, and genitalia are involved with or without skin manifestation. The distribution is symmetrical, involving the extremities, particularly the dorsal surfaces of the hands and feet. Attacks recur. The onset is abrupt and often accompanied by fever.

The cause of erythema multiforme exudativum is unknown. Suggested causes have been streptococcal infection and drugs. A viral cause is more likely in some cases. A preceding attack of herpes simplex is not uncommon. In the following case of erythema multiforme with target lesions herpes simplex virus was isolated and the complement-fixing antibodies showed a fourfold rise.

\section{CAse History}

A man of 30 was admitted to Lord Mayor Treloar Hospital on 8 March 1963 with a three-days history of ulcers in the mouth, difficulty in swallowing, and soreness of the eyes. He described two similar attacks, both lasting three weeks, the first in February 1957, the second in August 1961. None of the attacks was preceded by herpes simplex.
On examination his temperature was $102^{\circ}$ F. $\left(38.9^{\circ}\right.$ C.). Both conjunctivae were injected. He had red patches on the soft palate and fauces, and superficial ulceration of the tongue with oedema. There were erythematous raised patches, round in shape, on the anterior aspect of both legs. They did not itch and did not develop into bullae or vesicles. These lesions were rather trivial compared with those in the oral cavity. Four days after admission he complained of soreness of the penis, which on examination showed superficial ulceration of the glans. There was no urethral discharge. The erythematous lesions slowly regressed with symptomatic treatment and fever subsided in six days.

On admission his haemoglobin was $95 \%$; E.S.R. $13 \mathrm{~mm}$. in the hour ; white blood count $13,800 / \mathrm{c} . \mathrm{mm}$. (polymorphs $80 \%$, lymphocytes $15 \%$, monocytes $2 \%$, eosinophils $3 \%$ ) ; blood urea $31 \mathrm{mg} . / 100$ ml.; total proteins $7.4 \mathrm{~g} . / 100 \mathrm{ml}$. (albumin $2.85 \mathrm{~g}$., globulins $4.55 \mathrm{~g}$.); alpha-2- and gamma-globulin raised. The throat swab did not show any Vincent's organisms and the culture grew normal throat flora. On virus culture of the swab from throat lesions on 13 March herpes simplex was isolated. The complement-fixing antibodies were present at a dilution of $1 / 80$ on 11 March and at a dilution of $1 / 320$ on 21 March.

\section{Discussion}

The virus of herpes simplex is probably more widely distributed and more constantly present in man than any other virus. The virus has adapted itself to its human host and has achieved a highly successful parasitism, for over $60 \%$ of people in this country are infected with this virus and remain carriers throughout life.

Herpes simplex infection presents in different forms, such as herpes labialis, genital herpes, herpetic stomatitis, herpetic meningo-encephalitis, herpetic keratoconjunctivitis, and 